PROCEEDINGS OF THE

AMERICAN MATHEMATICAL SOCIETY

Volume 138, Number 8, August 2010, Pages 2803-2814

S 0002-9939(10)10365-7

Article electronically published on March 26, 2010

\title{
SCHATTEN(-HERZ) CLASS EXTENDED CESÀRO OPERATORS ON BERGMAN SPACES IN THE UNIT BALL OF $\mathbf{C}^{n}$
}

\author{
ZHANGJIAN HU AND XIAOMIN TANG \\ (Communicated by Marius Junge)
}

\begin{abstract}
For a large class of weights $\varphi$, we characterize the holomorphic symbols $g$, for which the extended Cesàro operators $T_{g}$ acting on the weighted Bergman space $A_{\varphi}^{2}(\mathbf{B})$ are in the Schatten (or Schatten-Herz) class in the unit ball of $\mathbf{C}^{n}$.
\end{abstract}

\section{INTRODUCTION}

Let $\mathbf{B}$ and $\partial \mathbf{B}$ be the open unit ball and the unit sphere in $\mathbf{C}^{n}$, and let $v$ and $\sigma$ be the normalized Lebesgue measure on $\mathbf{B}$ and $\partial \mathbf{B}$, respectively. When $n=1$, we denote the unit disc by $\mathbf{D}$.

A positive continuous function $\varphi$ on $[0,1)$ is called normal if there are two constants $b>a>-1$ such that

$$
\frac{\varphi(r)}{(1-r)^{a}} \downarrow 0, \quad \quad \frac{\varphi(r)}{(1-r)^{b}} \uparrow \infty
$$

as $r \rightarrow 1^{-}$. Given $\varphi$, we extend it to $\mathbf{B}$ by setting $\varphi(z)=\varphi(|z|)$, and we may assume that it is normalized so that $\int_{\mathbf{B}} \varphi(z) d v(z)=1$. The space $L_{\varphi}^{2}(\mathbf{B})$ is the space of all Lebesgue measurable functions $f$ on $\mathbf{B}$ for which

$$
\|f\|_{2, \varphi}=\left\{\int_{\mathbf{B}}|f(z)|^{2} \varphi(z) d v(z)\right\}^{\frac{1}{2}}<\infty .
$$

Let $H(\mathbf{B})$ be the family of all holomorphic functions on $\mathbf{B}$. The space

$$
A_{\varphi}^{2}(\mathbf{B})=L_{\varphi}^{2}(\mathbf{B}) \cap H(\mathbf{B})
$$

is called the weighted Bergman space. It is a closed subspace of $L_{\varphi}^{2}(\mathbf{B})$. Hence, $A_{\varphi}^{2}(\mathbf{B})$ is a Hilbert space with the inner product

$$
\langle f, g\rangle=\int_{\mathbf{B}} f(z) \overline{g(z)} \varphi(z) d v(z) .
$$

Received by the editors April 2, 2009 and, in revised form, November 5, 2009.

2010 Mathematics Subject Classification. Primary 47B38; Secondary 47B35, 32A36.

Key words and phrases. Extended Cesàro operator, Schatten class, Schatten-Herz class, Bergman space.

This project is supported by the National Natural Science Foundation of China (Nos. 10771064, 10971063), the Natural Science Foundation of Zhejiang Province (Nos. Y7080197, D7080080) and the Innovation Team Foundation of the Department of Education of the Zhejiang Province (No. T200924). 
For $g \in H(\mathbf{B})$, let $\Re g(z)=\sum_{j=1}^{n} z_{j} \frac{\partial g(z)}{\partial z_{j}}$ be the radial derivative of $g$. In [Hu02], the extended Cesàro operator on $A_{\varphi}^{2}(\mathbf{B})$ was defined. Precisely, given $g \in H(\mathbf{B})$, we define the extended Cesàro operator $T_{g}$ with the symbol $g$ as

$$
T_{g}(f)(z)=\int_{0}^{1} f(t z) \Re g(t z) \frac{d t}{t}, \quad f \in H(\mathbf{B}), \quad z \in \mathbf{B} .
$$

In the case that $n=1$,

$$
T_{g}(f)(z)=\int_{0}^{z} f(z) g^{\prime}(z) d z .
$$

The operator $T_{g}$ has attracted many mathematician's attention; for example, see AS95, AC01, Hu02, Hu04, Xi04, Ta07, LS08 and the references therein. In the case of one variable, A. Aleman and A. G. Siskakis proved in AS97 that $T_{g}$ is bounded (or compact) on $A_{\varphi}^{2}(\mathbf{D})$ if and only if $g$ is a Bloch function (or a little Bloch function). For the weight $\varphi(r)=(1-r)^{\alpha}$ with $\alpha>-1$ they also prove that $T_{g}$ is in the Schatten class $S_{p}$ with $p>1$ if and only if $g$ is in the Besov space and $T_{g}$ does not belong to $S_{p}$ with $0<p \leq 1$ unless $g$ is constant.

Recently in [LLP05], M. Loaiza, M. López-García and S. Pérez-Esteva introduced the concept of Shatten-Herz class Toeplitz operators. Given a finite Borel measure $\mu$ on $\mathbf{B}$, the Toeplitz operator $T(\mu)$ is defined on $A_{\varphi}^{2}(\mathbf{B})$ by

$$
T(\mu) f(z)=\int_{\mathbf{B}} f(w) K_{\varphi}(z, w) d \mu(w),
$$

where $K_{\varphi}(z, w)$ is the reproducing kernel of $A_{\varphi}^{2}(\mathbf{B})$. Following [LLP05, given $0<p, q<\infty, T(\mu)$ is said to be of the Schatten-Herz class $S_{p, q}\left(\right.$ write $T(\mu) \in S_{p, q}$ ) if $T\left(\mu \chi_{k}\right) \in S_{p}$ for each $k=0,1,2, \cdots$, and the $S_{p}$-norm sequence $\left\{\left\|T\left(\mu \chi_{k}\right)\right\|_{S_{p}}\right\}_{k=0}^{\infty}$ belongs to $l^{q}$, where $A_{k}=\left\{z \in \mathbf{B}: 1-\frac{1}{2^{k}} \leq|z|<1-\frac{1}{2^{k+1}}\right\}$ and $\chi_{k}$ is the characteristic function of $A_{k}$.

It will be shown (see Theorem 2.2 below) that $T_{g}^{*} T_{g}$ is a Toeplitz operator on $A_{\varphi}^{2}(\mathbf{B})$, so it is natural to give the following definition.

Definition 1.1. Let $0<p, q<\infty$ and $g \in H(\mathbf{B})$. The extended Cesàro operator $T_{g}$ is said to be of the Schatten-Herz class $S_{p, q}$, denoted by $T_{g} \in S_{p, q}$, if $T_{g}^{*} T_{g} \in S_{\frac{p}{2}}, \frac{q}{2}$.

The purpose of the present work is to characterize those symbols $g \in H(\mathbf{B})$ for which the induced extended Cesàro operators $T_{g} \in S_{p}\left(\right.$ or $\left.T_{g} \in S_{p, q}\right)$ on $A_{\varphi}^{2}(\mathbf{B})$. Our results are new even in the one-variable case. Here are the main results of this paper.

Theorem 1.1. Suppose $g \in H(\mathbf{B})$ and $0<p<\infty$. Then $T_{g} \in S_{p}$ if and only if

$$
\int_{\mathbf{B}}|\Re g(z)|^{p}\left(1-|z|^{2}\right)^{p-(n+1)} d v(z)<\infty .
$$

Moreover,

$$
\left\|T_{g}\right\|_{S_{p}} \simeq\left(\int_{\mathbf{B}}|\Re g(z)|^{p}\left(1-|z|^{2}\right)^{p-(n+1)} d v(z)\right)^{\frac{1}{p}} .
$$

Theorem 1.2. Suppose $g \in H(\mathbf{B})$ and $0<p, q<\infty$. Then $T_{g} \in S_{p, q}$ if and only if

$$
\int_{0}^{1} M_{p}^{q}(\Re g, r)\left(1-r^{2}\right)^{q-\left(n \frac{q}{p}+1\right)} d r<\infty
$$


Furthermore,

$$
\left\|T_{g}\right\|_{S_{p, q}} \simeq\left(\int_{0}^{1} M_{p}^{q}(\Re g, r)\left(1-r^{2}\right)^{q-\left(n \frac{q}{p}+1\right)} d r\right)^{\frac{1}{q}} .
$$

Here, $M_{p}(\Re g, r)=\left\{\int_{\partial \mathbf{B}}|\Re g(r \xi)|^{p} d \sigma(\xi)\right\}^{\frac{1}{p}}$.

In this paper, $C$ will always stand for positive constants whose value may change from line to line but not depend on the functions in $H(\mathbf{B})$. The expression $A \simeq B$ means $C^{-1} A \leq B \leq C A$.

\section{SOME PRELIMINARIES}

First, we exhibit some preliminary results, which will not only be used in the proof of the main results but also have their own interest.

Theorem 2.1. Given a normal weight $\varphi$ on $[0,1)$, set

$$
\phi(w)=\frac{1}{|w|^{2 n}} \int_{|w|}^{1} \frac{\int_{r}^{1} t^{2 n-1} \varphi(t) d t}{r} d r .
$$

Then the inner product on $A_{\varphi}^{2}(\mathbf{B})$ can be expressed as

$$
\langle f, g\rangle=f(0) \overline{g(0)}+4 \int_{\mathbf{B}} \Re f(w) \overline{\Re g(w)} \phi(w) d v(w) .
$$

Proof. If $f$ and $g$ are holomorphic in the unit disc $\mathbf{D} \subset \mathbf{C}$ and continuous on $\overline{\mathbf{D}}$, then

$$
\int_{0}^{2 \pi} f\left(e^{i \theta}\right) \overline{g\left(e^{i \theta}\right)} \frac{d \theta}{2 \pi}=f(0) \overline{g(0)}+2 \int_{\mathbf{D}} f^{\prime}(z) \overline{g^{\prime}(z)} \log \frac{1}{|z|} d m(z) .
$$

Here, $d m$ is the Lebesgue measure on D. Then, Prop. 1.4.7 in Ru80] implies, for $f, g \in H(\mathbf{B}) \cap C(\overline{\mathbf{B}})$, that

$$
\int_{\partial \mathbf{B}} f(\xi) \overline{g(\xi)} d \sigma(\xi)=f(0) \overline{g(0)}+2 \int_{\partial \mathbf{B}} d \sigma(\xi) \int_{\mathbf{D}} \Re f(z \xi) \overline{\Re g(z \xi)} \frac{1}{|z|^{2}} \log \frac{1}{|z|} d m(z) .
$$

Since

$$
\begin{aligned}
& \int_{\partial \mathbf{B}} d \sigma(\xi) \int_{\mathbf{D}} \Re f(z \xi) \overline{\Re g(z \xi)} \frac{1}{|z|^{2}} \log \frac{1}{|z|} d m(z) \\
= & \int_{\partial \mathbf{B}} d \sigma(\xi) \int_{0}^{1} \log \frac{1}{r} \frac{d r}{r} \int_{0}^{2 \pi} \Re f\left(r e^{i \theta} \xi \overline{\Re g\left(r e^{i \theta} \xi\right)} \frac{d \theta}{\pi}\right. \\
= & \int_{0}^{1} \log \frac{1}{r} \frac{d r}{r} \int_{\partial \mathbf{B}} d \sigma(\xi) \int_{0}^{2 \pi} \Re f\left(e^{i \theta} \cdot r \xi\right) \overline{\Re g\left(e^{i \theta} \cdot r \xi\right)} \frac{d \theta}{\pi} \\
= & 2 \int_{0}^{1} \log \frac{1}{r} \frac{d r}{r} \int_{\partial \mathbf{B}} \Re f(r \xi) \overline{\Re g(r \xi)} d \sigma(\xi) \\
= & \frac{1}{n} \int_{\mathbf{B}} \Re f(z) \overline{\Re g(z)} \log \frac{1}{|z|} \frac{d v(z)}{|z|^{2 n}},
\end{aligned}
$$

(2.2) gives

$$
\int_{\partial \mathbf{B}} f(\xi) \overline{g(\xi)} d \sigma(\xi)=f(0) \overline{g(0)}+\frac{2}{n} \int_{\mathbf{B}} \Re f(z) \overline{\Re g(z)} \log \frac{1}{|z|} \frac{d v(z)}{|z|^{2 n}} .
$$


This and [Ru80] yield, for $f, g \in A_{\varphi}^{2}(\mathbf{B})$,

$$
\begin{aligned}
& \int_{\mathbf{B}} f(z) \overline{g(z)} \varphi(z) d v(z) \\
= & 2 n \int_{0}^{1} r^{2 n-1} \varphi(r) d r \int_{\partial \mathbf{B}} f(r \xi) \overline{g(r \xi)} d \sigma(\xi) \\
= & 2 n \int_{0}^{1} r^{2 n-1} \varphi(r) d r\left[f(0) \overline{g(0)}+\frac{2}{n} \int_{\mathbf{B}} \Re f(r z) \overline{\Re g(r z)} \log \frac{1}{|z|} \frac{d v(z)}{|z|^{2 n}}\right] \\
= & f(0) \overline{g(0)}+4 \int_{0}^{1} r^{2 n-1} \varphi(r) d r \int_{\mathbf{B}} \Re f(r z) \overline{\Re g(r z)} \log \frac{1}{|z|} \frac{d v(z)}{|z|^{2 n}} .
\end{aligned}
$$

Taking the variable transformation $w=r z$, we have

$$
\begin{aligned}
& \int_{0}^{1} r^{2 n-1} \varphi(r) d r \int_{\mathbf{B}} \Re f(r z) \overline{\Re g(r z)} \log \frac{1}{|z|} \frac{d v(z)}{|z|^{2 n}} \\
= & \int_{0}^{1} r^{2 n-1} \varphi(r) d r \int_{|w|<r, z \in \mathbf{B}} \Re f(w) \overline{\Re g(w)} \frac{r^{2 n}}{|w|^{2 n}} \log \frac{r}{|w|} \frac{d v(w)}{r^{2 n}} \\
= & \int_{\mathbf{B}} \Re f(w) \overline{\Re g(w)} \frac{1}{|w|^{2 n}} d v(w) \int_{|w|}^{1} r^{2 n-1} \varphi(r) \log \frac{r}{|w|} d r .
\end{aligned}
$$

Because

$$
\begin{aligned}
\int_{|w|}^{1} r^{2 n-1} \varphi(r) \log \frac{r}{|w|} d r & =\int_{|w|}^{1} \log \frac{r}{|w|}\left[\frac{d}{d r}\left(-\int_{r}^{1} t^{2 n-1} \varphi(t) d t\right)\right] d r \\
& =\int_{|w|}^{1} \frac{\int_{r}^{1} t^{2 n-1} \varphi(t) d t}{r} d r
\end{aligned}
$$

(2.4) gives

$$
\begin{aligned}
& \int_{0}^{1} r^{2 n-1} \varphi(r) d r \int_{\mathbf{B}} \Re f(r z) \overline{\Re g(r z)} \log \frac{1}{|z|} \frac{d v(z)}{|z|^{2 n}} \\
= & \int_{\mathbf{B}} \Re f(w) \overline{\Re g(w)} \frac{1}{|w|^{2 n}}\left(\int_{|w|}^{1} \frac{\int_{r}^{1} t^{2 n-1} \varphi(t) d t}{r} d r\right) d v(w) .
\end{aligned}
$$

Putting (2.5) into (2.3), we obtain (2.1). The proof is complete.

In particular, in the very special case that $n=1$ and $\varphi(r)=(\alpha+1)\left(1-r^{2}\right)^{\alpha}$ with $\alpha>-1$, the equality (2.1) is just (5.1) of AS97.

We are now going to exhibit the relation between the extended Cesàro operator and the Toeplitz operator. Recall that, given a finite Borel measure $\mu$ on $\mathbf{B}$, the Toeplitz operator $T(\mu)$ on $A_{\varphi}^{2}(\mathbf{B})$ is defined by

$$
T(\mu) f(z)=\int_{\mathbf{B}} f(w) K_{\varphi}(z, w) d \mu(w)
$$

where $K_{\varphi}(z, w)$ is the reproducing kernel of $A_{\varphi}^{2}(\mathbf{B})$. Toeplitz operators have been extensively studied by many authors; see Lu87, Zh88, Zh07, CKN07, Lu08. We also need Bloch functions. A holomorphic function $g$ on $\mathbf{B}$ is said to be a Bloch function if $\sup _{z \in \mathbf{B}}|\Re g(z)|\left(1-|z|^{2}\right)<\infty$.

Theorem 2.2. Suppose $g$ is a Bloch function on $\mathbf{B}$. Then the operator $T_{g}^{*} T_{g}$ is a Toeplitz operator $T(\mu)$ induced by the measure $d \mu(w)=4|\Re g(w)|^{2} \phi(w) d v(w)$. 
Proof. Since $g$ is a Bloch function, by $\mathrm{Hu} 04, T_{g}$ is bounded on $A_{\varphi}^{2}(\mathbf{B})$; hence the adjoint $T_{g}^{*}$ is also bounded. Notice also that $\Re\left(T_{g} f\right)(z)=f(z) \Re g(z)$. Then, for $f \in A_{\varphi}^{2}(\mathbf{B})$, applying Theorem 2.1 we get

$$
\begin{aligned}
T_{g}^{*} T_{g} f(z) & =\left\langle T_{g}^{*} T_{g} f, K_{\varphi}(\cdot, z)\right\rangle \\
& =\left\langle T_{g} f, T_{g} K_{\varphi}(\cdot, z)\right\rangle \\
& =4 \int_{\mathbf{B}} f(w) \Re g(w) \overline{K_{\varphi}(w, z)} \cdot \overline{\Re g(w)} \phi(w) d v(w) \\
& =\int_{\mathbf{B}} f(w) K_{\varphi}(z, w) d \mu(w),
\end{aligned}
$$

where $d \mu=4|\Re g(w)|^{2} \phi(w) d v(w)$. The proof is complete.

\section{Schatten class extended Cesàro operators}

In this section we will give the proof of Theorem 1.1. Before doing this we need some more preliminary results. Let $H$ be a separable Hilbert space with the inner product $\langle\cdot, \cdot\rangle$, and let $T$ be a positive compact operator on $H$. There exists (see [Zh90] for example) an orthonormal set $\left\{e_{k}\right\}$ in $H$ and a sequence $\left\{\lambda_{k}\right\}$ decreasing to 0 such that

$$
T x=\sum_{k} \lambda_{k}\left\langle x, e_{k}\right\rangle e_{k}
$$

for all $x \in H$. The numbers $\lambda_{k}$ are called the singular values of $T$. For $p>0, T$ is said to be in the Schatten class $S_{p}$ if

$$
\|T\|_{S_{p}}=\left(\sum_{k} \lambda_{k}^{p}\right)^{\frac{1}{p}}<\infty .
$$

More generally, a compact (not necessarily positive) operator $T$ on $H$ belongs to $S_{p}$ if the positive operator $|T|=\left(T^{*} T\right)^{\frac{1}{2}}$ belongs to $S_{p}$. In this case, define $\|T\|_{S_{p}}=$ $\||T|\|_{S_{p}}$. For more details, see [Zh90].

Let $\beta(\cdot, \cdot)$ denote the Bergman metric on $\mathbf{B}$. The Bergman ball $D(z, \rho)$ with center $z \in \mathbf{B}$ and radius $\rho>0$ is defined as $D(z, \rho)=\{w \in \mathbf{B}: \beta(z, w)<\rho\}$. Set $d \lambda(z)=\frac{d v(z)}{\left(1-|z|^{2}\right)^{n+1}}$. It is easy to see that

$$
|D(z, \rho)| \simeq\left(1-|z|^{2}\right)^{n+1}, \quad 1-|w| \simeq 1-|z| \text { for } w \in D(z, \rho) .
$$

Given a finite positive Borel measure $\mu$ on $\mathbf{B}$ and $\rho>0$, define the averaging function $\hat{\mu}_{\rho}$ by $\widehat{\mu}_{\rho}(z)=\frac{\mu(D(z, \rho))}{\int_{D(z, \rho)} \varphi(w) d v(w)}$. Since $\varphi$ is normal, by (1.1) it is easy to check that

$$
\widehat{\mu}_{\rho}(z) \simeq \frac{\mu(D(z, \rho))}{\varphi(z)\left(1-|z|^{2}\right)^{n+1}}
$$

(see [Hu02]). The following lemma is in [LH10].

Lemma 3.1. Suppose $\mu$ is a finite positive Borel measure on $\mathbf{B}$ and $0<p<\infty$. Then $T_{\mu} \in S_{p}$ if and only if $\widehat{\mu}_{\rho} \in L^{p}(\lambda)$ for any (or some) $\rho>0$. In this case,

$$
\left\|T_{\mu}\right\|_{S_{p}} \simeq\left\|\widehat{\mu}_{\rho}\right\|_{L^{p}(\lambda)} .
$$

Now we are in the position to give the proof of Theorem 1.1. 
Proof of Theorem 1.1. We prove the sufficiency first. Suppose $0<p<\infty$ and that $g \in H(\mathbf{B})$ satisfies

$$
\int_{\mathbf{B}}|\Re g(z)|^{p}\left(1-|z|^{2}\right)^{p-(n+1)} d v(z)<\infty .
$$

This means that $g$ is in the $p$-th Besov space, and hence $g$ is in the little Bloch space. By [Hu04, $T_{g}$ is compact on $A_{\varphi}^{2}(\mathbf{B})$. Applying Theorem 2.2 and Lemma 3.1 , we need only prove that $\hat{\mu}_{\rho} \in L^{\frac{p}{2}}(\mathbf{B}, d \lambda)$ for $\mu(w)=4|\Re g(w)|^{2} \phi(w) d v(w)$. Now we fix $\rho>0$ so that

$$
\bigcup_{|z| \leq \frac{1}{2}} D(z, \rho) \subset\left\{z:|z| \leq \frac{3}{4}\right\} \quad \text { and } \bigcup_{|z| \geq \frac{1}{2}} D(z, \rho) \subset\left\{z:|z| \geq \frac{1}{4}\right\} .
$$

Since $\varphi$ is integrable on the interval $[0,1)$,

$$
\phi(w)=\frac{1}{|w|^{2 n}} \int_{|w|}^{1} \frac{\int_{r}^{1} t^{2 n-1} \varphi(t) d t}{r} d r \leq C \frac{1}{|w|^{2 n}} \log \frac{1}{|w|} .
$$

For fixed $\zeta \in \partial \mathbf{B}$, set $g_{\zeta}(\lambda)=g(\lambda \zeta)$ for $\lambda \in \mathbf{D}$. From 6.4.4 in [Ru80] we know that $\Re g(\lambda \zeta)=\lambda g_{\zeta}^{\prime}(\lambda)$. Hence

$$
\begin{aligned}
\sup _{z \in \mathbf{B},|z| \leq \frac{3}{4}} \frac{|\Re g(z)|}{|z|} & =\sup _{\lambda \in \mathbf{D},|\lambda| \leq \frac{3}{4}, \zeta \in \partial \mathbf{B}} \frac{\left|\lambda g_{\zeta}^{\prime}(\lambda)\right|}{|\lambda|}=\sup _{\lambda \in \mathbf{D},|\lambda|=\frac{3}{4}, \zeta \in \partial \mathbf{B}}\left|g_{\zeta}^{\prime}(\lambda)\right| \\
& =C \sup _{\lambda \in \mathbf{D},|\lambda|=\frac{3}{4}, \zeta \in \partial \mathbf{B}}\left|\lambda g_{\zeta}^{\prime}(\lambda)\right|=C \sup _{z \in \mathbf{B},|z|=\frac{3}{4}}|\Re g(z)| .
\end{aligned}
$$

Therefore, by (3.1) and the plurisubharmonicity of $|\Re g(z)|^{p}$,

$$
\begin{aligned}
\sup _{|z| \leq \frac{1}{2}} \widehat{\mu}_{\rho}(z) & \leq C \int_{|z| \leq \frac{3}{4}}|\Re g(z)|^{2} \phi(z) d v(z) \\
& \leq C \sup _{|z| \leq \frac{3}{4}} \frac{|\Re g(z)|^{2}}{|z|^{2}} \int_{|z| \leq \frac{3}{4}} \frac{1}{|z|^{2 n-2}} \log \frac{1}{|z|} d v(z) \\
& \leq C \sup _{|z|=\frac{3}{4}}|\Re g(z)|^{2} \\
& \leq C\left\{\int_{\frac{1}{2}<|w|<\frac{7}{8}}|\Re g(w)|^{p} d v(w)\right\}^{\frac{2}{p}} \\
& \leq C\left\{\int_{\mathbf{B}}|\Re g(w)|^{p}\left(1-|w|^{2}\right)^{p-(n+1)} d v(w)\right\}^{\frac{2}{p}} .
\end{aligned}
$$

For $\frac{1}{4} \leq r<1$, by definition (1.1) and [Hu04],

$$
\frac{\int_{r}^{1} t^{2 n-1} \varphi(t) d t}{r} \simeq \int_{r}^{1} \varphi(t) d t \simeq \varphi(r)(1-r) \text { and } \varphi(w) \simeq \varphi(z) \text { for } w \in D(z, \rho) .
$$


Then, for $|z|>\frac{1}{2}$ we have

$$
\begin{aligned}
\hat{\mu}_{\rho}(z)^{\frac{p}{2}} & \simeq\left\{\frac{\int_{D(z, \rho)}|\Re g(w)|^{2} \varphi(w)\left(1-|w|^{2}\right)^{2} d v(w)}{\int_{D(z, \rho)} \varphi(w) d v(w)}\right\}^{\frac{p}{2}} \\
& \simeq\left\{\left(1-|z|^{2}\right)^{1-n} \int_{D(z, \rho)}|\Re g(w)|^{2} d v(w)\right\}^{\frac{p}{2}} \\
& \leq C\left(1-|z|^{2}\right)^{p} \sup _{w \in D(z, \rho)}|\Re g(w)|^{p} \\
& \leq C\left(1-|z|^{2}\right)^{p} \frac{1}{\mid D(z, 2 \rho)) \mid} \int_{D(z, 2 \rho)}|\Re g(w)|^{p} d v(w) \\
& \leq C\left(1-|z|^{2}\right)^{p-(n+1)} \int_{D(z, 2 \rho)}|\Re g(w)|^{p} d v(w) .
\end{aligned}
$$

Therefore,

$$
\begin{aligned}
& \int_{|z| \geq \frac{1}{2}}\left|\hat{\mu}_{\rho}(z)\right|^{\frac{p}{2}} d \lambda(z) \\
\leq & C \int_{|z|>\frac{1}{2}}\left(\int_{D(z, 2 \rho)}|\Re g(w)|^{p} d v(w)\right)\left(1-|z|^{2}\right)^{p-2(n+1)} d v(z) \\
\leq & C \int_{\mathbf{B}}\left(\int_{D(z, 2 \rho)}|\Re g(w)|^{p} d v(w)\right)\left(1-|z|^{2}\right)^{p-2(n+1)} d v(z) \\
= & C \int_{\mathbf{B}}|\Re g(w)|^{p}\left(\int_{\mathbf{B}} \chi_{D(z, 2 \rho)}(w)\left(1-|z|^{2}\right)^{p-2(n+1)} d v(z)\right) d v(w) \\
\leq & C \int_{\mathbf{B}}|\Re g(w)|^{p}\left(1-|w|^{2}\right)^{p-(n+1)} d v(w) .
\end{aligned}
$$

Combine (3.3) and (3.5) to obtain

$$
\begin{aligned}
\int_{\mathbf{B}}\left|\hat{\mu}_{\rho}(z)\right|^{\frac{p}{2}} d \lambda(z) & =\left\{\int_{|z|<\frac{1}{2}}+\int_{|z| \geq \frac{1}{2}}\right\}\left|\hat{\mu}_{\rho}(z)\right|^{\frac{p}{2}} d \lambda(z) \\
& \leq C \int_{\mathbf{B}}|\Re g(z)|^{p}\left(1-|z|^{2}\right)^{p-(n+1)} d v(z) .
\end{aligned}
$$

This gives the desired estimate:

$$
\left\|T_{g}\right\|_{S_{p}} \leq C\left\{\int_{\mathbf{B}}|\Re g(z)|^{p}\left(1-|z|^{2}\right)^{p-(n+1)} d v(z)\right\}^{\frac{1}{p}} .
$$

Now we prove the necessity. Suppose $\left\|T_{g}\right\|_{S_{p}}<\infty$. We claim that

$$
\hat{\mu}_{\rho}(z) \geq C|\Re g(z)|^{2}\left(1-|z|^{2}\right)^{2} .
$$

In fact, if $|z| \leq \frac{1}{2}$ and $w \in D(z, \rho)$, it is easy to check that $\phi(w) \geq C$. Hence, for $|z| \leq \frac{1}{2}$

$$
\hat{\mu}_{\rho}(z) \geq \frac{C}{\varphi(z)|D(z, \rho)|} \int_{D(z, \rho)}|\Re g(w)|^{2} d v(w) \geq C|\Re g(z)|^{2}\left(1-|z|^{2}\right)^{2} .
$$


If $|z|>\frac{1}{2}$, by (3.4) and the plurisubharmonicity of $|\Re g(z)|^{2}$,

$$
\hat{\mu}_{\rho}(z) \geq\left(1-|z|^{2}\right)^{1-n} \int_{D(z, \rho)}|\Re g(w)|^{2} d v(w) \geq C|\Re g(z)|^{2}\left(1-|z|^{2}\right)^{2} .
$$

Then (3.7) follows from these two inequalities. Now (3.7) and Lemma 3.1 yield

$$
\int_{\mathbf{B}}|\Re g(z)|^{p}\left(1-|z|^{2}\right)^{p-(n+1)} d v(z) \leq C \int_{\mathbf{B}}\left|\hat{\mu}_{\rho}(z)\right|^{\frac{p}{2}} d \lambda(z) \leq C\left\|T_{g}\right\|_{S_{p}}^{p} .
$$

Combine this and (3.6) to obtain the desired estimate:

$$
\left\|T_{g}\right\|_{S_{p}} \simeq\left\{\int_{\mathbf{B}}|\Re g(z)|^{p}\left(1-|z|^{2}\right)^{p-(n+1)} d v(z)\right\}^{\frac{1}{p}} .
$$

The proof is complete.

The Besov space $B_{p}$ consists of all functions $f \in H(\mathbf{B})$ for which

$$
\|f\|_{B_{p}}=\left\{\int_{\mathbf{B}}|\Re g(z)|^{p}\left(1-|z|^{2}\right)^{p-(n+1)} d v(z)\right\}^{\frac{1}{p}}<\infty .
$$

It is easy to verify that $B_{p}$ contains constants only if $0<p \leq n$. In terms of the space $B_{p}$, Theorem 1.1 can be reformulated as below.

Corollary 3.1. Suppose $g \in H(\mathbf{B})$ and $0<p<\infty$. Then for $p>n, T_{g} \in S_{p}$ if and only if $g \in B_{p}$; and for $0<p \leq n, T_{g}$ does not belong to $S_{p}$ unless $g$ is constant. While for $n<p<\infty,\left\|T_{g}\right\|_{S_{p}} \simeq\|g\|_{B_{p}}$.

\section{Schatten-Herz Class extended Cesàro operators}

In this section we prove Theorem 1.2. First, we recall some more about SchattenHerz class Toeplitz operators. We decompose $\mathbf{B}$ into the disjoint union of annuli $A_{k}=\left\{z \in \mathbf{B}: r_{k} \leq|z|<r_{k+1}\right\}$, where $r_{k}=1-2^{-k}, k=0,1,2, \cdots$. For each $k$, set $\chi_{k}=\chi_{A_{k}}$, the characteristic function of $A_{k}$. For $0<p, q<\infty$, the Herz space $\mathcal{K}_{q}^{p}(\lambda)$ consists of measurable function $f$ for which

$$
\|f\|_{\mathcal{K}_{q}^{p}(\lambda)}=\left\|\left\{\left\|f \chi_{k}\right\|_{L^{p}(\lambda)}\right\}\right\|_{l^{q}} .
$$

Given $\mu$ a finite Borel measure, let $\mu \chi_{k}$ stand for the restriction of $\mu$ to $A_{k}$. Given $0<p, q<\infty$, the induced Toeplitz operator $T(\mu)$ is said to be of the SchattenHerz class $S_{p, q}$, denoted by $T(\mu) \in S_{p, q}$, if $T\left(\mu \chi_{k}\right) \in S_{p}$ for each $k$ and the sequence $\left\{\left\|T\left(\mu \chi_{k}\right)\right\|_{S_{p}}\right\}_{k=0}^{\infty}$ belongs to $l^{q}$. The $S_{p, q}$-norm of $T(\mu)$ is defined by $\|T(\mu)\|_{S_{p, q}}=$ $\left\|\left\{\left\|T\left(\mu \chi_{k}\right)\right\|_{S_{p}}\right\}\right\|_{l q}$. For more details on Herz spaces and the Schatten-Herz class Toeplitz operators, see [LLP05, CKL07, CKN07, HY99].

The following lemma comes from LH10.

Theorem 4.1. Suppose $\mu$ is a finite positive Borel measure on $\mathbf{B}$ and $0<p, q<\infty$. Then $T(\mu) \in S_{p, q}$ if and only if $\widehat{\mu}_{\rho} \in \mathcal{K}_{q}^{p}(\lambda)$ for any (or some) $\rho>0$. Furthermore,

$$
\|T(\mu)\|_{S_{p, q}} \simeq\left\|\widehat{\mu}_{\rho}\right\|_{\mathcal{K}_{q}^{p}(\lambda)} .
$$

Proof of Theorem 1.2. By Theorem 2.2 and Theorem 4.1, $T_{g} \in S_{p, q}$ if and only if

$$
\hat{\mu}_{\rho} \in \mathcal{K}_{\frac{q}{2}}^{\frac{p}{2}}(\lambda) \quad \text { and } \quad\left\|T_{g}\right\|_{S_{p, q}} \simeq\left\|\widehat{\mu}_{\rho}\right\|_{\mathcal{K}_{\frac{q}{2}}^{\frac{p}{2}}(\lambda)},
$$


where $\rho>0$ is fixed and small enough. Thus, we need only prove that

$$
\sum_{k=0}^{\infty}\left\{\int_{A_{k}} \hat{\mu}_{\rho}(z)^{\frac{p}{2}} d \lambda(z)\right\}^{\frac{q}{p}} \simeq \int_{0}^{1} M_{p}^{q}(\Re g, r)\left(1-r^{2}\right)^{q-\left(n \frac{q}{p}+1\right)} d r
$$

For each $k \geq 1$, by $(3.7)$ and the fact that $M_{p}(\Re g, t)$ is increasing in $(0,1)$ we have

$$
\begin{aligned}
\int_{A_{k}} \hat{\mu}_{\rho}(z)^{\frac{p}{2}} d \lambda(z) & \geq C \int_{A_{k}}|\Re g(z)|^{p}\left(1-|z|^{2}\right)^{p} d \lambda(z) \\
& \simeq 2^{k[(n+1)-p]} \int_{r_{k}}^{r_{k+1}} r^{2 n-1} d r \int_{\partial \mathbf{B}}|\Re g(r \xi)|^{p} d \sigma(\xi) \\
& \geq C 2^{k[(n+1)-p-1]} M_{p}^{p}\left(\Re g, r_{k}\right), \quad k=1,2, \cdots
\end{aligned}
$$

Therefore, with $(1-t) \simeq 2^{-k}$ for $t \in\left(r_{k-1}, r_{k}\right)$ in mind,

$$
\begin{aligned}
\sum_{k=0}^{\infty}\left\{\int_{A_{k}} \hat{\mu}_{\rho}(z)^{\frac{p}{2}} d \lambda(z)\right\}^{\frac{q}{p}} & \geq C \sum_{k=1}^{\infty} 2^{\frac{q k}{p}(n-p)} M_{p}^{q}\left(\Re g, r_{k}\right) \\
& \geq C \sum_{k=1}^{\infty} \int_{r_{k-1}}^{r_{k}}(1-t)^{q-n \frac{q}{p}-1} M_{p}^{q}(\Re g, t) d t \\
& =C \int_{0}^{1}(1-t)^{q-\left(n \frac{q}{p}+1\right)} M_{p}^{q}(\Re g, t) d t .
\end{aligned}
$$

This gives one direction estimate of (4.1). Now we deal with the other direction. Since

$$
\inf _{|k-l| \geq 2} \beta\left(A_{k}, A_{l}\right)>C>0
$$

we can fix $\rho>0$ so that $\widetilde{A_{k}}=\bigcup_{z \in A_{k}} D(z, 2 \rho)$ satisfies

$$
\widetilde{A_{k}} \subseteq\left\{z \in \mathbf{B}: r_{k-1} \leq|z|<r_{k+2}\right\}=A_{k-1} \cup A_{k} \cup A_{k+1},
$$

here $A_{-1}=A_{0}$. For $z \in \mathbf{B}$, with some $w_{0} \in \overline{D(z, \rho)}$ we have

$$
\begin{aligned}
{\left[\int_{D(z, \rho)}|\Re g(w)|^{2} d v(w)\right]^{\frac{p}{2}} } & \leq\left[|D(z, \rho)| \max _{w \in \overline{D(z, \rho)}}|\Re g(w)|^{2}\right]^{\frac{p}{2}} \\
& \leq C\left(1-|z|^{2}\right)^{(n+1) \frac{p}{2}}\left|\Re g\left(w_{0}\right)\right|^{p} \\
& \leq C \frac{\left(1-|z|^{2}\right)^{(n+1) \frac{p}{2}}}{\left|D\left(w_{0}, \rho\right)\right|} \int_{D\left(w_{0}, \rho\right)}|\Re g(u)|^{p} d v(u) \\
& \leq C\left(1-|z|^{2}\right)^{(n+1)\left(\frac{p}{2}-1\right)} \int_{D(z, 2 \rho)}|\Re g(w)|^{p} d v(w) .
\end{aligned}
$$


For $k \geq 1$, combine (3.4) and (4.3) to obtain

$$
\begin{aligned}
\int_{A_{k}} & \hat{\mu}_{\rho}(z)^{\frac{p}{2}} d v(z) \\
& \leq C \int_{A_{k}}\left(1-|z|^{2}\right)^{(1-n) \frac{p}{2}}\left(\int_{D(z, \rho)}|\Re g(w)|^{2} d v(w)\right)^{\frac{p}{2}} d v(z) \\
& \leq C \int_{A_{k}}\left(1-|z|^{2}\right)^{(1-n) \frac{p}{2}+(n+1)\left(\frac{p}{2}-1\right)}\left(\int_{D(z, 2 \rho)}|\Re g(w)|^{p} d v(w)\right) d v(z) \\
& \leq C \int_{A_{k}}\left(1-|z|^{2}\right)^{p-(n+1)}\left(\int_{\widetilde{A_{k}}}|\Re g(w)|^{p} \chi_{D(z, 2 \rho)}(w) d v(w)\right) d v(z) \\
& \leq C \int_{\widetilde{A_{k}}}|\Re g(w)|^{p}\left(\int_{A_{k}}\left(1-|z|^{2}\right)^{p-(n+1)} \chi_{D(z, 2 \rho)}(w) d v(z)\right) d v(w) \\
& \leq C \int_{\widetilde{A_{k}}}\left(1-|w|^{2}\right)^{p}|\Re g(w)|^{p} d v(w) .
\end{aligned}
$$

Therefore,

$$
\begin{aligned}
& \left\{\int_{A_{k}} \hat{\mu}_{\rho}(z)^{\frac{p}{2}} d \lambda(z)\right\}^{\frac{q}{p}} \\
\leq & C\left(1-r_{k}\right)^{-(n+1) \frac{q}{p}}\left\{\int_{A_{k}} \hat{\mu}_{\rho}(z)^{\frac{p}{2}} d v(z)\right\}^{\frac{q}{p}} \\
\leq & C\left(1-r_{k}\right)^{-(n+1) \frac{q}{p}}\left\{\int_{\widehat{A_{k}}}\left(1-|w|^{2}\right)^{p}|\Re g(w)|^{p} d v(w)\right\}^{\frac{q}{p}} \\
\leq & C\left(1-r_{k}\right)^{-(n+1) \frac{q}{p}}\left\{\int_{r_{k-1}}^{r_{k+2}}\left(1-r^{2}\right)^{p} M_{p}^{p}(\Re g, r) d r\right\}^{\frac{q}{p}} \\
\leq & C\left(1-r_{k}\right)^{-(n+1) \frac{q}{p}+(p+1) \frac{q}{p}} M_{p}^{q}\left(\Re g, r_{k+2}\right), \quad k=1,2, \cdots .
\end{aligned}
$$

Meanwhile, by (3.2) we get

$$
\left\{\int_{A_{0}} \hat{\mu}_{\rho}(z)^{\frac{p}{2}} d \lambda(z)\right\}^{\frac{q}{p}} \leq C\left\{\int_{\frac{1}{2} \leq|z| \leq \frac{7}{8}}|\Re g(z)|^{p} d v(z)\right\}^{\frac{q}{p}} \leq C M_{p}^{q}\left(\Re g, r_{3}\right) .
$$

(4.4) and (4.5) yield

$$
\begin{aligned}
\sum_{k=0}^{\infty}\left\{\int_{A_{k}} \hat{\mu}_{\rho}(z)^{\frac{p}{2}} d \lambda(z)\right\}^{\frac{q}{p}} & \leq C \sum_{k=1}^{\infty}\left(1-r_{k}\right)^{q-n \frac{q}{p}} M_{p}^{q}\left(\Re g, r_{k+2}\right) \\
& \leq C \sum_{k=1}^{\infty} \int_{r_{k+2}}^{r_{k+3}}(1-t)^{q-n \frac{q}{p}-1} M_{p}^{q}(\Re g, t) d t \\
& \leq C \int_{0}^{1}(1-t)^{q-\left(n \frac{q}{p}+1\right)} M_{p}^{q}(\Re g, t) d t .
\end{aligned}
$$

From this and (4.2) we obtain (4.1). The proof is complete. 
Notice that, for $0<p \leq n$ and $g \in H(\mathbf{B})$,

$$
\int_{0}^{1} M_{p}^{q}(\Re g, r)\left(1-r^{2}\right)^{q-\left(n \frac{q}{p}+1\right)} d r<\infty
$$

implies that $g$ is a constant. Similar to Corollary 3.1 we have the following at once.

Corollary 4.1. Suppose $g \in H(\mathbf{B})$ and $0<p, q<\infty$. Then for $p>n, T_{g} \in S_{p, q}$ if and only if $\int_{0}^{1} M_{p}^{q}(\Re g, r)\left(1-r^{2}\right)^{q-\left(n \frac{q}{p}+1\right)} d r<\infty$; and for $0<p \leq n, T_{g}$ does not belong to $S_{p, q}$ unless $g$ is a constant. While for $n<p<\infty,\left\|T_{g}\right\|_{S_{p, q}} \simeq$ $\left\{\int_{0}^{1} M_{p}^{q}(\Re g, r)\left(1-r^{2}\right)^{q-\left(n \frac{q}{p}+1\right)} d r\right\}^{\frac{1}{q}}$.

\section{REFERENCES}

[AC01] A. Aleman, J. Cima, An integral operator on $H^{p}$ and Hardy's inequality, J. Anal. Math., 85 (2001), 157-176. MR 1869606 (2002k:30068)

[AS95] A. Aleman, A. G. Siskakis, An integral operator on $H^{p}$, Complex Variables, 28 (1995), 149-158. MR.1700079 (2000d:47050)

[AS97] A. Aleman, A. G. Siskakis, Integration operators on Bergman spaces, Indiana University Math. J., 46 (1997), 337-356. MR1481594 (99b:47039)

[CKL07] B. R. Choe, H. Koo, Y. J. Lee, Positive Schatten(-Herz) class Toeplitz operators on the half-space, Potential Analysis, 27 (2007), 73-100. MR2314190(2008k:31007)

[CKN07] B. R. Choe, H. Koo, K. Na, Positive Toeplitz operators of Schatten-Herz type, Nagoya Math. J., 185 (2007), 31-62. MR2301457 (2007m:47057)

[Hu02] Z. J. Hu, Extended Cesàro operators on mixed norm spaces, Proc. Amer. Math. Soc., 131 (2003), 2171-2179. MR1963765 (2004a:47030)

[Hu04] Z. J. Hu, Extended Cesàro operators on Bergman spaces, J. Math. Anal. Appl., 296 (2004), 435-454. MR2075175 (2005e:47087)

[HY99] E. Hernández, D. Yang, Interpolation of Herz spaces and applications, Math. Nachr, 205 (1999), 69-87. MR.1709163 (2000e:46035)

[LH10] X. F. Lv, Z. J. Hu, Schatten(-Herz) classes Toeplitz operators on weighted Bergman spaces. (Chinese), Acta Math. Sinica, 53(4) (2010), in press.

[LLP05] M. Loaiza, M. López-García, S. Pérez-Esteva, Herz classes and Toeplitz operators in the disk, Integr. Equ. Oper. Theory, 53 (2005), 287-296. MR2187174 (2006g:47041)

[LS08] S. X. Li, S. Stević, Compactness of Riemann-Stieltjes operators between $F(p, q, s)$ spaces and $\alpha$-Bloch spaces, Publ. Math. Debrecen, 72 (2008), 1-2. MR2376863 (2008m:47048)

[Lu87] D. Luecking, Trace ideal criteria for Toeplitz operators, J. Funct. Anal., 73 (1987), 345-368. MR899655 (88m:47046)

[Lu08] D. H. Luecking, Finite rank Toeplitz operators on the Bergman space, Proc. Amer. Math. Soc., 136 (5) (2008), 1717-1723. MR2373601 (2008m:47038)

[Ru80] W. Rudin, Function Theory in the Unit Ball of $\mathbf{C}^{n}$, New York, Springer-Verlag, 1980. MR601594 (82i:32002)

[Ta07] X. M. Tang, Extended Cesàro operators between Bloch-type spaces in the unit ball of $\mathbf{C}^{n}$, J. Math. Anal. Appl., 326 (2007), 1199-1211. MR2280974 (2007j:47064)

[Xi04] J. Xiao, Riemann-Stieltjes operators on weighted Bloch and Bergman spaces of the unit ball, J. London Math. Soc. (2), 70 (2004), 199-214. MR2064758 (2005c:47044)

[Zh88] K. H. Zhu, Positive Toeplitz operators on weighted Bergman spaces of bounded symmetric domains, J. Operator Theory, 20 (1988), 329-357. MR.1004127|(92f:47022) 
[Zh90] K. H. Zhu, Operator theory in function spaces, Springer-Verlag, New York, 1990. MR:1074007 (92c:47031)

[Zh07] K. H. Zhu, Schatten class Toeplitz operators on weighted Bergman spaces of the unit ball, New York J. Math., 13 (2007), 299-316. MR.2357717 (2008g:47058)

Department of Mathematics, Huzhou Teachers College, Huzhou, Zhejiang 313000 , People's Republic of China

E-mail address: huzj@hutc.zj.cn

Department of Mathematics, University of Science and Technology of China, Hefei, Anhui 230026, People's Republic of China - and - Department of Mathematics, Huzhou Teachers College, Huzhou, Zhejiang 313000, People's Republic of China

E-mail address: txm@hutc.zj.cn 